Stoffe darin nicht fehlen, so ist bei Stagnation und W ärme eine Fäulniss, wobei Schwefelwasserstoffgas auftritt, die nothwendige Folge.

Jena, den 2. April 1869.

\title{
Ueber den Polyhalit im Steinsalze zu Stassfurt
}

\author{
vou Professor E. Reichardt in Jena. *)
}

Der Polyhalit im Steinsalze zu Stassfurt wurde von nir zuerst in denjenigen Salzschichten nachgewiesen, welche zwischen dem eigentlichen massiven und sehr reinen Steinsalze und den Kalisalzen - bunten, bitteren Salzen - liegen und so den Uebergang vermitteln. Bi sch of hat diese Abtheilung als Yolyhalitregion bezeichnet. Hier findet sich dieses Mineral in verhältnissmässig nicht starken Schnüren zwischen dem Steinsalze, dasselbe in ziemlich regelmässiger Richtung durchsetzend. Sowohl Bischof's wie meine Analysen haben die Zusammensetzung des Polyhalites erwiesen.

In neuester Keit hatte Herr Bergmeister Schöne, Director des Anhaltinischen Salzwerkes Stassfurt, die Güte, mir ein Vorkommen zu übersenden, welches in stärkeren Stücken sporadisch als Einmengung auftritt. Man fand es in derselben Region, wo der Kaïnit, den Carnallit zurückdrängend, sich ausbreitet, sowohl im Kainit, wie im Carnallit. Nach den in Stassfurt unternommenen Untersuchungen war man schon veranlasst, Polyhalit zu vermuthen. Beide Stïcke, welche ich erhielt, hatten eine dichte, weisse, körnige Beschaffenheit, zeigten hier und da kleine Partien Carnallit und wurden getrennt der Untersuchung unterworfen, welche mein Assistent, Herr Dreykorn, ausfihrte:

Spec. Gew, bei $20^{\circ} \mathrm{C} .=2,69 ; \mathrm{Bisch}$ of fand bei den Polyhalitschnüren $2,72, \mathrm{~N}$ a u man n giebt für Polyhalit überhaupt 2,7 bis 2,8 an:

Die chemische Untersuchung ergab in 100 Theilen der Substanz :

\footnotetext{
*) Vom Hr. Verfasser als Separatabdruck crhalten. Die Red.
} 


\begin{tabular}{lrr} 
& \multicolumn{1}{c}{ I. } & \multicolumn{1}{c}{ II. } \\
Wasser, bei $110^{\circ} \mathrm{C}$. entweichend & 0,29 & 0,77 \\
Glühverlust & 8,10 & 6,38 \\
Kali & 7,32 & 10,73 \\
Natron & 3,69 & 3,14 \\
Kalk & $\mathbf{1 8 , 9 2}$ & 17,38 \\
Talkerde & 7,57 & 7,42 \\
Schwefelsäure & 52,53 & 52,15 \\
Chlor & 0,61 & 1,18 \\
Kieselsäure & - & 0,12 \\
& 99,03 & $99,27$.
\end{tabular}

Bei dem Austrocknen der Substanz bei $110^{\circ} \mathrm{C}$. tral schon saure Reaction hervor, welche sich bei weiterem Glï. hen verstärkte und so das bekannte Verhalten von $\mathrm{MgCl}$ erwies. Der Glühverlust muss daher einen Ueberschus: ergeben, sobald derselbe als Wasser in Rechnung gestellt wird.

Auf Salze berechnet ergiebt diess, unter Bindung des $\mathrm{Cl}$ an $\mathrm{Mg}$ :

I. II.

\begin{tabular}{lrr} 
Schwefelsaures Kali & 13,53 & $\mathbf{1 9 , 8 4}$ \\
\multicolumn{1}{c}{ Natron } & 8,45 & 7,35 \\
Schwefelsauren Kalk & 45,95 & $\mathbf{4 2 , 4 9}$ \\
Schwefelsaure Talkerde & $\mathbf{2 1 , 8 1}$ & 20,27 \\
Chlormagnium & 0,79 & $\mathbf{1 , 5 8}$ \\
Kieselsäure & - & 0,12 \\
Wasser, bei $110^{\circ} \mathrm{C}$. entweichend & 0,29 & 0,77 \\
Glühverlust & 8,10 & 6,38 \\
\hline & 98,92 & $98,80$.
\end{tabular}

Gefunden wurden bei 1. 52,53 Proc. $\mathrm{SO}^{3}$, die Berechnung der Salze erfordert 52,54; bei II. gefunden 52,15, berechnet $=51,74$.

Die Formel des Polyhalites ${\mathrm{KO}, \mathrm{SO}^{3}}^{3} \mathrm{MgO}, \mathrm{SO}^{3}+$ $2\left(\mathrm{CaO}, \mathrm{SO}^{3}\right)+2 \mathrm{HO}$ verlangt: 


$\begin{array}{lr}\mathrm{KO}, \mathrm{SO}^{3} & 28,87 \\ {\mathrm{MgO}, \mathrm{SO}^{3}} & 20,01 \\ \mathrm{CaO}, \mathrm{SO}^{3} & 45,16 \\ \mathrm{HO} & \mathbf{5 , 9 6 .}\end{array}$

Berechnet man das oben angegebene $\mathrm{NaO}, \mathrm{SO}^{3}$ auf $\mathrm{KO}, \mathrm{SO}^{3}$ und addirt das Resultat dem andern zu, so ergiebt $\mathrm{I}$. in Summe 26,48, II. 28,84 Proc. $\mathrm{KO}, \mathrm{SO}^{3}$, letztere Zahl stimmt mit derjenigen der berechneten procentischen Zusammensetzung nahezu überein.

Die Formel des Polyhalites wurde von H. Rose festgestellt, Analysen von demselben ergaben:

Rose. Reichardt. Bischof.

berechn.

I. II. III. IV. V. VI. VII.

$\begin{array}{lllllllll}\mathrm{CaO} \mathrm{SO}^{3} & 42,29 & 45,62 & 41,72 & 43,44 & 42,64 & 45,95 & 42,49 & 45,16\end{array}$ $\begin{array}{lllllllll}\mathrm{NgO}, \mathrm{SO}^{3} & 18,27 & 18,97 & 17,80 & 20,56 & 19,76 & 21,81 & 20,27 & 20,01\end{array}$

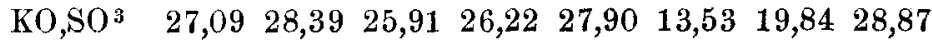
$\begin{array}{lllllllll}\mathrm{NaO}, \mathrm{SO}^{3} & 2,60 & 0,61 & 0 & - & - & 8,45 & 7,35 & -\end{array}$ $\begin{array}{lllllllll}\mathrm{NaCl} & 1,38 & 0,31 & 0,41 & - & 3,49 & - & - & - \\ \mathrm{MgCl} & - & - & - & 0,58 & - & 0,79 & 1,58 & -\end{array}$ $\begin{array}{llllllll}\mathrm{SO}^{3} & 0,27 & 0,32 & - & - & - & - & 0,12\end{array}$

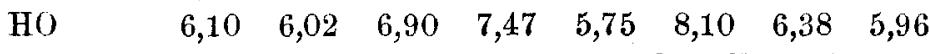
$98,00 \quad 100,24 \quad 92,74 \quad 98,27 \quad 99,5 \quad 498,63 \quad 98,03 \quad 100,00$

I. Breitstängeliger Polyhalit angeblich von Hallein; II. ziegelrother, dünn und grobschaliger von Aussee; III. grauer Polyhalit von Vic in Lothringen; IV. und V. Polyhalit von Stassfurt aus der sogenannten Polyhalitregion; VI. and VII. die beiden neuen Untersuchungen (I. und II.) von oben.

Die oben schon gegebenen Zahlen bei Annahme der Substitution von Natron und Kali, verglichen mit der neugebotenen Zusammenstellung ergeben unzweifelhaft Polyhalit, in welchem ein nicht unwesentlicher Theil des Kali's durch $\mathrm{Na}$ tron vertreten ist, sonst stimmen alle Resultate mit denjenigen der Analysen anderer Chemiker überein.

Die Division mit den Aequivalentenzahlen bei dem neuen Vorkommen ergiebt: 
14 Ueber den angeblichen Dextringehalt der essharen Kastanien.

\begin{tabular}{|c|c|c|c|c|c|c|}
\hline & 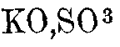 & $\mathrm{NaO}, \mathrm{S}$ & $\mathrm{MgO}$ & & $a c$ & \\
\hline I. & 1,3 & : & 3,05 & : & 5,7 & : \\
\hline & 2,2 & : & $3, \%$ & : & 6,1 & . \\
\hline
\end{tabular}

Einfache äquivalente Beziebungen zwischen $\mathrm{KO}^{\mathrm{SO} \mathrm{S}^{3}}$ un $\mathrm{NaO}, \mathrm{SO}^{3}$ ergeben sich darans nicht, dagegen stimmen di

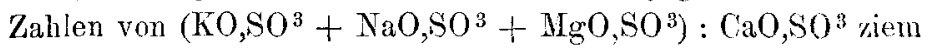
lich überein bei I. wie $5,35: 5,7$,

, II. $, 6,5: 6,1$.

$\mathrm{KO}, \mathrm{SO}^{3}+\mathrm{NaO}, \mathrm{SO}^{3}$ verhält sich zu $\mathrm{MgO}, \mathrm{SO}^{3}$, wie

$\begin{array}{cccc}\text { I. } & 2,3 & : & 3,05 \\ \text { II. } & 3,3 & : & 3,30\end{array}$

Es sind Schwankungen, wie sie von H. Rose sogleicl bei den ersten Untersuchungen der Polyhalite und der Be grïndung der Fornel erhalten wurden und welche immer au die mehrfache Vertretung hinzeigen. In dem vorliegender Falle erstreckt sich diese besonders auf die Alkalien.

Unaufgeklärt ist jedoch das mit Ausnahme der Analyser von II. und V. überall gleichmässig auftretende Resultat dass die Summe der Bestandtheile um 1-2 Procente Ver. luste ergiebt. IV. ist von mir selbst untersucht worden: VI. und VII. von Dreykorn; derselbe hat mehrfache Controle - Versuche eingelegt, so dass die Genanigkeit der Untersuchung eine nur wünschenswerthe ist.

\section{Ueber den angeblichen Dextringehalt der essbaren Kastanien,}

von H. Ludwig.

In der Fortsetzung des Handbuchs der organischen Chemie von L. Gmelin, 4. Bd., 1. Abth. S. 631 (hearb. v. Lr. Karl Kraut) findet sich in Betreff des Vorkommens des Dextrins die Angabe: "in den echten Kastanien zu 22,8 bis 23,3 Proc., " nach Albini (Wiener Acad. Ber. 13, 502.). Nach diesen Berichten vom Jahre 1854 enthalten die entschäl- 